\title{
How to tackle congestion in acute heart failure
}

\author{
Pieter Martens $^{1,2}$ and Wilfried Mullens ${ }^{1,3}$
}

\begin{abstract}
${ }^{1}$ Department of Cardiology, Ziekenhuis Oost-Limburg, Genk; ${ }^{2}$ Doctoral School for Medicine and Life Sciences, Hasselt University, Diepenbeek; ${ }^{3}$ Biomedical Research Institute, Faculty of Medicine and Life Sciences, Hasselt University, Diepenbeek, Belgium
\end{abstract}

Received: November 15, 2017 Accepted: December 3, 2017

\section{Correspondence to}

\section{Wilfried Mullens, M.D.}

Department of Cardiology,

Ziekenhuis Oost-Limburg, Schiepse Bos 6, 3600 Genk, Belgium

Tel: $+32-89-327160$

Fax: $+32-89-327918$

E-mail: Wilfried.Mullens@zol.be
Acute heart failure is a common complication of chronic heart failure and is associated with a high risk for subsequent mortality and morbidity. In $90 \%$ of case acute heart failure is the resultant of congestion, a manifestation of fluid build-up due to increased filling pressures. As residual congestion at discharge following an acute heart failure episodes is one of the strongest predictors of poor outcome, the goal of therapy should be to resolve congestion completely. Important to comprehend is that increased cardiovascular filling pressures are not solely the resultant of intravascular volume excess but can also be induced by a decreased venous capacitance. This review article focusses on the pathophysiology, diagnoses, and treatment of congestion in acute heart failure. A clear distinction is made between states of volume overload (intravascular volume excess) or volume redistribution (decreased venous capacitance) contributing to congestion in acute heart failure.

Keywords: Heart failure; Congestion

\section{INTRODUCTION}

Acute heart failure (AHF) represents a seminal event in the disease trajectory of chronic heart failure (CHF) [1] and is associated with increased risks of re-hospitalization and mortality [2]. The costs of frequent readmissions to the hospital create a significant economic burden on society [3]. In total, $90 \%$ of AHF cases that require hospitalization are due to worsening congestion, and only a minority of these cases are due to low cardiac output [4]. Many drugs have been developed in the last decade to reduce the burden of congestion during AHF [5-10]. However, numerous trials have failed to significantly decrease the risks of hospitalization for heart failure and all-cause mortality, suggesting that it is difficult to influence long-term outcomes using transitory medication. Many trials have also indicated that congestion is often unresolved when patients are discharged. AHF therapies have not changed dramatically in the past few years, and diuretics and vasodilators remain the main- stays of treatment $[11,12]$. Although cardiologists have confidence in the efficacy of diuretics and vasodilators, there is a lack of information on how to titrate these therapies [11,12].

This review provides an overview of the pathophysiology of congestion in heart failure, focusing on effective strategies for alleviating congestion. It emphasizes the importance of achieving complete decongestion, because residual congestion is the most significant predictor of hospital readmission and postdischarge mortality [13].

\section{UNDERSTANDING CONGESTION}

\section{What is congestion?}

Congestion is defined as the signs and symptoms of extracellular fluid accumulation, instigated by an increase in left-sided cardiac filling pressure [14]. This definition recognizes that poor cardiac function is a prerequisite 
for developing congestion. However, numerous other organs play a role in the development of congestion, including the splanchnic veins and the interstitial and endothelial tissues. The use of implantable hemodynamic monitoring devices have significantly contributed to our understanding of how CHF develops into AHF [15]. One of the earliest changes is a small pressure increase in the pulmonary artery or right side of the heart, which takes place over an extended time period. This often occurs weeks in advance of the patient presenting at a hospital with AHF. Once filling pressures significantly increase, symptoms of functional deterioration occur that often result in patients going to the emergency department. This process is often described as the transition between hemodynamic congestion and clinical congestion. At this stage, clinical congestion might lead to pulmonary edema with desaturation, myocardial ischemia, a progressive decline in kidney function, cerebral changes, and an increased risk of arrhythmia. In this way, a disease that normally progresses slowly is transformed into a life-threatening condition.

\section{What mechanisms produce congestion?}

The progressive accumulation of sodium and water is often considered the major reason why clinical congestion leads to AHF. Indeed, heart failure is frequently characterized as compensatory neurohormonal activation that leads to chronic sodium and water retention. Alterations in both the proximal and distal parts of the nephron play a central role in the enhanced avidity for sodium that occurs in the kidneys during heart failure [16]. Approximately $65 \%$ of this retained sodium is stored in the extracellular compartment [17], which consists of both an intravascular compartment and the interstitium. Sodium is retained iso-osmotically, with approximately $75 \%$ retained in the interstitium and only $25 \%$ retained in the intravascular compartment. Therefore, changes in total blood volume are always associated with a 3 -fold increase in interstitial volume. In addition, changes in total blood volume are not evenly distributed across the arterial and venous systems. The venous system contains up to $70 \%$ of the total blood volume, most of which is in the high capacitance splanchnic veins [18]. These splanchnic veins can accommodate a $65 \%$ increase in blood volume without changes in central filling pressures [19]. Therefore, a patient's weight must have increased significantly in the weeks prior to hospitalization for heart failure, if central filling pressures have increased solely by iso-osmotic sodium retention. Indeed, studies on patients who present with AHF and signs of weight gain have shown that the blood-volume compartment expands by approximately $40 \%$. Assuming an average blood volume of $5 \mathrm{~L}$, this would mean an expansion of 1.9 L. However, because approximately $75 \%$ of the sodium is retained in the interstitium, there is an additive retention of $5.85 \mathrm{~L}$ in the interstitium [20]. Follow-up studies on these patients with decongestion indicated that most of the diuresed volume originated in the interstitium. By contrast, several studies have found that nearly $50 \%$ of patients presenting with AHF did not have significant weight gain prior to decompensation [21]. Therefore, chronic progressive volume expansion is not the only reason for congestion.

Changes in the capacitance of the splanchnic veins can also contribute to the development of congestion [22]. The splanchnic system can act as a volume buffer to protect the cardiovascular system from blood loss. By decreasing their capacitance, mediated by activation of the sympathetic nervous system, the splanchnic veins can augment their cardiac preload and maintain cardiac output when blood volume is low [23]. However, these changes in venous capacitance probably also contribute to the development of congestion, particularly in patients who have not gained weight $[24,25]$. Therefore, both changes in total blood volume (volume overload) and venous capacitance (volume redistribution) can lead to congestion. Indeed, as of 2016, the European Society Guidelines for the treatment of acute and CHF recommend that clinicians differentiate between volume overload and volume redistribution when treating AHF. However, it is clear that the two mechanisms are not mutually exclusive and both may contribute to congestion in many patients with AHF (Fig. 1).

\section{The importance of resolving congestion completely} Irrespective of the mechanisms causing congestion, once it has developed, congestion is self-perpetuated in a variety of ways. Studies of selectively induced coronary-sinus congestion in dogs demonstrated a decrease in coronary perfusion and changes in myocardial ventricular stiffening with a downward and rightward shift in the end-systolic pressure volume relationship [26-28]. 


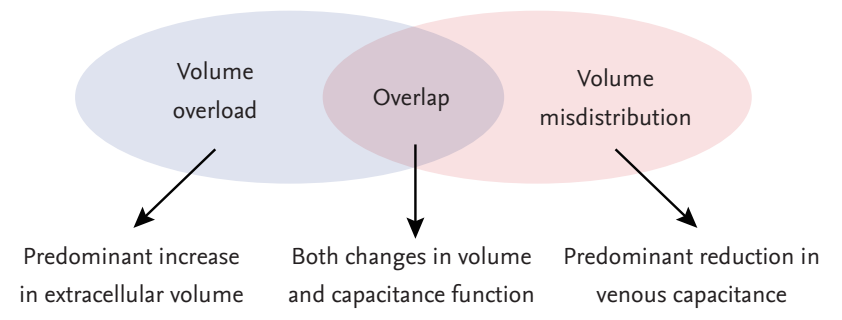

Figure 1. Relationship between intravascular volume and filling pressures in acute heart failure.

Changes in central venous pressure are also associated with worsening renal function (WRF), resulting in a drop in natriuresis [29-31]. Heart-rate variability data showed a shift from autonomic toward sympathetic nervous system control [32], which can further decrease splanchnic venous capacitance and increase preload [22]. In addition, splanchnic congestion results in increased intra-abdominal pressure, WRF, and ischemia [22,33]. Ischemia reduces the effectiveness of the intestinal barrier, leading to lipopolysaccharide-induced endotoxicity and increasing inflammation [22]. Colombo et al. [34] used a selective forearm-congestion model to demonstrate that transient congestion results in endothelial activation and promotes inflammation. The ensuing endothelial activation results in decreased arterial impedance and venous capacitance, which negatively influences afterload and preload in AHF. Therefore, congestion is self-amplifying, and complete decongestion should always be the goal when treating patients with this condition. It is not surprising that achieving decongestion in patients with AHF or those awaiting transplants for advanced heart failure is associated with improved outcomes. Indeed, residual congestion at discharge following hospitalization for AHF is the strongest predictor of subsequent readmission and cardiovascular mortality.

\section{EVALUATION OF THE CONGESTED PATIENT}

\section{Is the patient congested?}

Determining the presence of clinical congestion is often easy for the experienced physician. Clinical symptoms and signs of dyspnea, orthopnea, bendopnea, rales, the third heart sound, jugular venous distention, and pulmonary or peripheral edema all point to congestion.
When combined, these signs have high specificity for detecting increased cardiac filling pressures. However, even a combination of rales, elevated jugular venous distention, and edema only has a 58\% sensitivity for detecting an elevated pulmonary capillary wedge pressure. Therefore, auxiliary tests are often necessary to detect hemodynamic congestion, which lacks clear clinical signs or symptoms. The gold standard for assessing congestion remains right heart catheterization and direct measurements of right atrial and pulmonary capillary wedge pressure using a pulmonary artery catheter [35]. In most patients with heart failure, the right-sided and left-sided filling pressures are closely related [36]. Therefore, right-sided measurements can be used as a barometer for left-sided pressure in most patients. The Evaluation Study of Congestive Heart Failure and Pulmonary Artery Catheterization Effectiveness (ESCAPE) trial failed to demonstrate that direct measurements of filling pressures with tailored therapy and a pulmonary artery catheter were superior to clinically guided assessments of AHF patients [35]. As a result, the use of invasive tailored therapy to treat AHF has declined. However, a pulmonary artery catheter remains useful for patients with unclear hemodynamic profiles at baseline or uncertain hemodynamic responses following therapy (e.g., WRF, right-left mismatches, or worsening heart failure). Cardiac hemodynamics are now frequently assessed by non-invasive methods. Echocardiography provides a comprehensive non-invasive evaluation of cardiac hemodynamics [37]. Tables 1 and 2 provides an overview of the echo-derived parameters used to asses right-sided versus left-sided filling pressure. These echocardiographic parameters can also detect subclinical congestion. Some data have suggested that targeted decongestion, based on echocardiography, can reduce the risk for hospitalization for heart failure [38]. In addition to echocardiography, natriuretic peptides (NPs) are increasingly being used to assess cardiac filling pressures non-invasively, and are useful for diagnosing the presence of congestion and stratifying patients according to risk. However, their value in determining the success of decongestive therapy remains questionable [39]. Indeed, NP levels probably reflect atrial dimensions and are affected by changes in local geometry and stretch triggers, which are influenced by age, body mass index, and kidney function [39]. An additional problem is the lag times in 
Table 1. Right-sided assessment: sensitivity and specificity for variables predicting right atrial pressure $>7 \mathrm{mmHg}$

\begin{tabular}{lccl}
\hline & Sensitivity, \% & Specificity, \% & Setting assessment \\
\hline Clinical variable & & & \\
JVP $>7$ mmHg & 48 & 78 & Advanced chronic HF \\
\hline Jugular venous reflux & 50 & 75 & Advanced chronic HF \\
Hepatomegaly & 51 & 62 & Advanced chronic HF \\
Edema & 10 & 94 & Advanced chronic HF \\
Echocardiographic variable & & & \\
Collapse (<40\%) IVC & 12 & 27 & Advanced chronic HF \\
Inspiratory diameter IVC $<12 \mathrm{~mm}$ & 67 & 91 & Advanced chronic HF \\
\hline
\end{tabular}

JVP, jugular venous pressure; $\mathrm{HF}$, heart failure; IVC, inferior vena cava.

Table 2. Left-sided assessment: sensitivity and specificity for variables predicting pulmonary capillary wedge pressure $>18$ $\mathrm{mmHg}$

\begin{tabular}{|c|c|c|c|}
\hline & Sensitivity, \% & Specificity, \% & Setting assessment \\
\hline \multicolumn{4}{|l|}{ Clinical variable } \\
\hline Dyspnea & 50 & 73 & Advanced chronic HF \\
\hline Dyspnea on exertion & 66 & 52 & Advanced chronic HF \\
\hline Orthopnea & 66 & 47 & Advanced chronic HF \\
\hline $\mathrm{S}_{3}$ & 73 & 42 & Advanced chronic HF \\
\hline Rales & 13 & 90 & Advanced chronic HF \\
\hline \multicolumn{4}{|l|}{ Echocardiographic variable } \\
\hline E wave $>50 \mathrm{~cm} / \mathrm{sec}$ & 92 & 28 & Advanced chronic HF \\
\hline Lateral E/E'> 12 & 66 & 55 & Acute HF \\
\hline Lateral E/E' > 12 & 42 & 85 & Advanced chronic HF \\
\hline Deceleration time $<130 \mathrm{msec}$ & 81 & 80 & Advanced chronic HF \\
\hline Pulmonary vein $S / D<1$ & 83 & 72 & Advanced chronic HF \\
\hline Fixed interatrial septum & 100 & 97 & Advanced chronic HF \\
\hline
\end{tabular}

HF, heart failure; $\mathrm{S}_{3}$, third heart sound; $\mathrm{E}$ wave, peak velocity flow in early diastole; E/E', early filling velocity to early diastolic mitral annular velocity; S/D, systolic/diastolic ratio.

changes in NP levels following hemodynamic changes, as these levels equilibrate approximately 1 week after clinical stabilization [39].

\section{Does volume overload or volume redistribution predominate?}

Filling pressures are poor surrogates for intravascular volume because they are also affected by venous compliance. A meta-analysis of studies comparing central venous pressure with blood volume measurements indicated that only $2.5 \%$ of central venous pressure variability across the study population was explained by differences in blood volume. Therefore, the observation that nearly $50 \%$ of AHF patients had gained less than $1 \mathrm{~kg}$ in weight is unsurprising, because both volume overload (changes in volume) and volume redistribution (changes in venous compliance) can contribute to congestion. At present, no study has evaluated clinical or technical strategies for differentiating between these causes of AHF. However, significant weight gain during the week prior hospitalization for heart failure and peripheral edema suggests that expansion of the extracellular compartment, and therefore volume overload, has occurred. More recently, several AHF studies have examined the 
feasibility of using direct plasma volume measurements to address this problem. Miller and Mullan [20] measured the blood volume of 26 AHF patients with a clinical diagnosis of volume overload. The measurements showed that the plasma-volume compartment expanded to different degrees among AHF patients. However, $8 \%$ of the patients with a clinical diagnosis of volume overload actually had decreased plasma volumes [20] and may have had volume misdistribution rather than volume overload. These findings indicate that directly measuring plasma volume may be useful for differentiating among causes of congestion and for titrating therapies. However, at present, a detailed patient history that includes weight changes and clinical assessments of edema (e.g., peripheral edema, ascites, bowel edema) may be the most reliable method for differentiating between volume overload and redistribution [40].

\section{TREATMENT OF CONGESTION}

Because one of the strongest predictors of re-hospitalization and mortality in AHF patients is residual congestion on discharge from hospital, the goal of therapy should be to achieve complete decongestion [13]. In addition, once congestion has been resolved, it is important to prevent it from recurring. Although volume overload and volume redistribution can overlap in clinical practice, we propose that the emphasis of decongestive therapy should be on the prevailing mechanism of congestion.

\section{Tackling congestion with volume overload}

When volume overload is causing congestion, the goal of therapy should be to decrease extracellular sodium and water, bearing in mind that extracellular volume is found in both the interstitium and plasma [17]. In addition, the only physiological methods of removing excessive sodium and water are kidney-mediated natriuresis and diuresis [16]. Direct diuresis of the interstitium is not possible, and to decongest the interstitium, the Starling forces between the interstitium and the plasma compartment need to be reversed [41]. This is called 'plasma-refilling' and is achieved by decreasing hydrostatic capillary pressure and increasing oncotic capillary pressure of the plasma compartment, secondary to re- nal natriuresis and diuresis. To effectively tackle volume overload, a thorough understanding of the renal changes that cause increased sodium avidity in AHF patients is necessary. This information is beyond the scope of the present review, but has previously been described [16]. Loop diuretics remain the mainstay of decongestive therapy for volume overload. However, if diuretic efficacy is low, combinational diuretic therapy may be necessary to enhance the diuretic response and facilitate decongestion (Fig. 2) [42].

\section{Loop diuretics}

Loop diuretics are the mainstay of diuretic therapy for AHF, and almost $90 \%$ of patients in the ADHERE (Acute Decompensated Heart Failure National Registry) database were treated using intravenous loop diuretics [43]. In addition, in $63 \%$ of patients, loop diuretics are the sole drug therapy used to combat AHF [43]. Despite the ubiquitous use of loop diuretics, only one randomized controlled trial, the Diuretic Optimization Strategies Evaluation (DOSE) trial, has prospectively assessed their performance in treating AHF [44]. In the DOSE trial, 308 patients were randomly assigned low and high doses of diuretics $(2 \times 2)$, either continuously or in bolus form. No differences were observed between patients who had diuretics administered continuously compared to those who ingested them in bolus form. However, those patients receiving the higher dose of furosemide (median dose of $773 \mathrm{mg}$ vs. $358 \mathrm{mg}$ in 72 hours) showed a trend towards faster relief from dyspnea and significantly higher net fluid and weight losses. When the glomerular filtration rate is low, it is often necessary to increase the dose of loop diuretics. Recent research has also shown that early interventions using loop diuretics may be associated with improved outcomes in AHF patients [45]. However, treatment with loop diuretics may also be associated with neurohormonal activation and ionic disturbances and has never been shown to reduce mortality rates [46]. In addition, a significant number of patients treated with loop diuretics do not achieve decongestion, and it is unclear whether these patients would benefit from an increased dose or combination-diuretic therapy.

Combination-diuretic treatment and bail-out therapy If loop diuretics fail to relieve volume overload, there is 

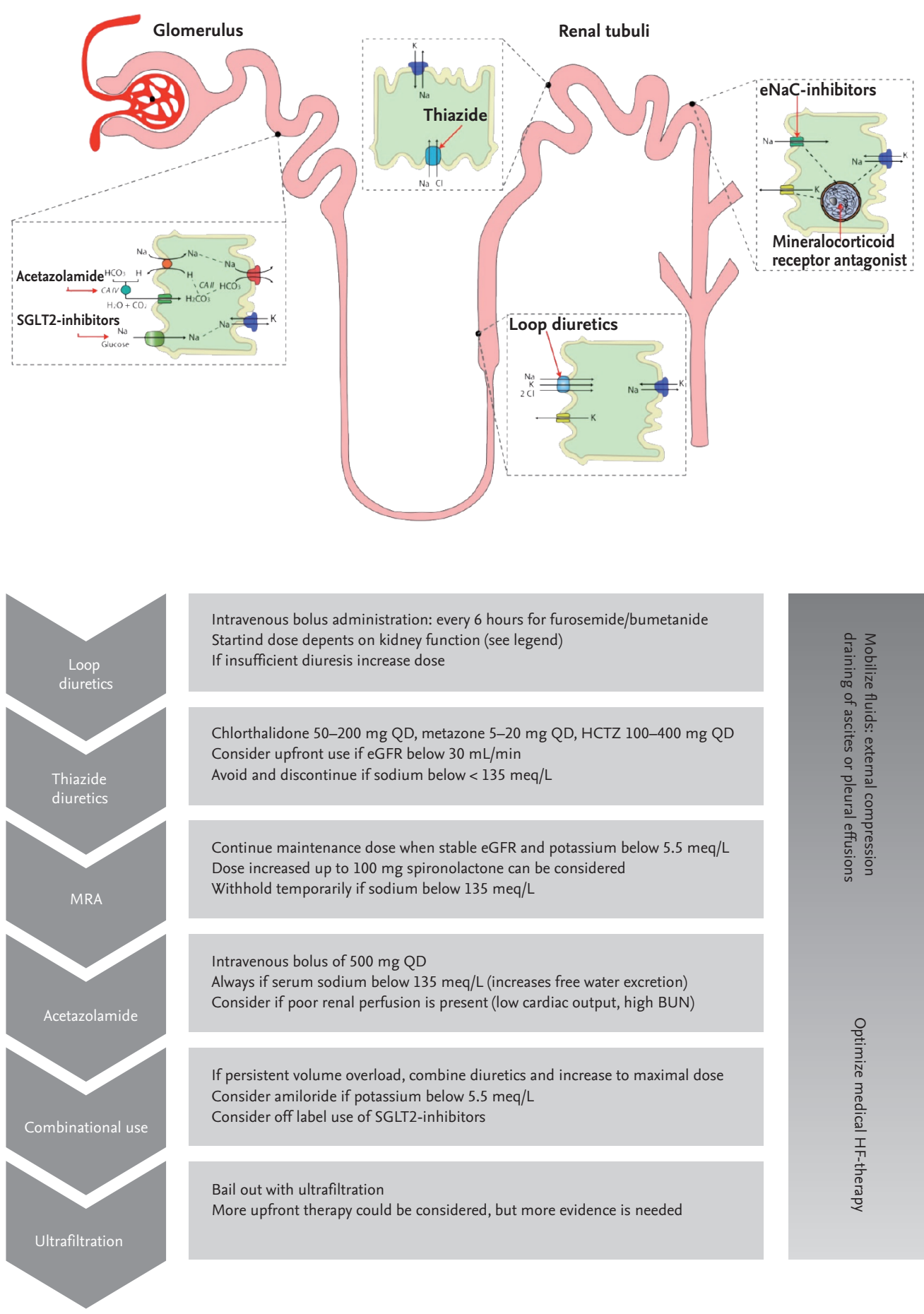

Intravenous bolus administration: every 6 hours for furosemide/bumetanide Startind dose depents on kidney function (see legend)

If insufficient diuresis increase dose

Chlorthalidone 50-200 mg QD, metazone 5-20 mg QD, HCTZ 100-400 mg QD

Consider upfront use if eGFR below $30 \mathrm{~mL} / \mathrm{min}$

Avoid and discontinue if sodium below $<135 \mathrm{meq} / \mathrm{L}$

Continue maintenance dose when stable eGFR and potassium below $5.5 \mathrm{meq} / \mathrm{L}$

Dose increased up to $100 \mathrm{mg}$ spironolactone can be considered

Withhold temporarily if sodium below $135 \mathrm{meq} / \mathrm{L}$

Intravenous bolus of $500 \mathrm{mg}$ QD

Always if serum sodium below $135 \mathrm{meq} / \mathrm{L}$ (increases free water excretion)

Consider if poor renal perfusion is present (low cardiac output, high BUN)

If persistent volume overload, combine diuretics and increase to maximal dose Consider amiloride if potassium below $5.5 \mathrm{meq} / \mathrm{L}$

Consider off label use of SGLT2-inhibitors

Bail out with ultrafiltration

More upfront therapy could be considered, but more evidence is needed

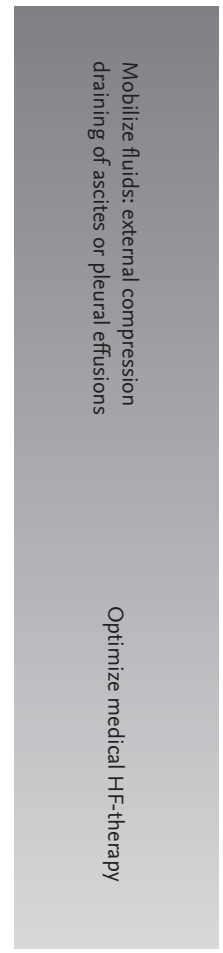

Figure 2. Targets for combinational diuretic therapy in volume overload. QD, once a day; HCTZ, hydrochlorothiazide; eGFR, estimated glomerular filtration rate; BUN, blood urea nitrogen; SGLT-2, sodium-glucose-linked transporter 2; MRA, mineralocorticoid receptor antagonist; HF, heart failure. 
little information to suggest the best alternative treatment. Indeed, changing the dose or using another loop diuretic are frequently suggested by practice guidelines [12]. Fig. 2 provides an overview of the targets within the kidney nephron and the potential strategies for enhancing natriuresis and diuresis. Thiazide diuretics are frequently used to treat patients who are resistant to loop diuretics [47]. Indeed, prolonged used of loop diuretics can result in intrinsic renal adaptations, including enhanced sodium avidity, which are largely mediated by thiazide-sensitive sodium chloride transporters [48]. One limitation of thiazides is that they reduce the kidneys' capacity for producing dilute urine. Therefore, they should be avoided by patients with hypotonic hyponatremia [49]. Mineralocorticoid receptor antagonists are an intrinsic part of CHF therapy; however, at higher doses they have natriuretic effects [50]. They are often used when loop diuretics induce renal potassium wasting. The recent ATHENA-HF (Aldosterone Targeted Neurohormonal Combined with Natriuresis Therapy in Heart Failure) trial failed to show that a high dose of spironolactone improved decongestion when administered with standard therapy and evaluated using changes in NP levels at 96 hours, compared to placebo [51]. Acetazolamide is an outdated diuretic that is still used to treat mountain sickness and glaucoma. One observational study found that when acetazolamide was used along with loop diuretics to treat AHF, the diuretic efficacy increased by $100 \mathrm{mg}$ of sodium per $40 \mathrm{mg}$ furosemide equivalents [52]. Currently, one small prospective study (NCTo1973335) is evaluating the effects of acetazolamide in AHF patients. Sodium-glucose-linked transporter 2 (SGLT-2) inhibitors also induce natriuresis and reduce plasma volume by inhibiting proximal nephron sodium reabsorption [53]. Two trials of diabetic patients with cardiovascular disease showed that SGLT-2 inhibitors were highly effective for decreasing the likelihood of hospitalization for heart failure [54,55]. Empagliflozine is currently being tested in stable heart failure patients with both reduced (NCT03057977) and preserved (NCT03057951) ejection fractions. However, its potential for treating patients with AHF is unclear. If congestion persists despite therapy with diuretics, bail-out therapy with ultrafiltration may be necessary. The use of ultrafiltration to treat AHF patients with persistent congestion is beyond the scope of this review, but has recently been reviewed in detail [56].

\section{When is volume overload completely resolved?}

One of the biggest problems in treating patients who have congestion with volume overload is knowing when euvolemia has been achieved. Retrospective analyses of the DOSE and CARESS (Clopidogrel and Aspirin for Reduction of Emboli in Symptomatic Carotid Stenosis) trials have indicated that even in patients with no edema or orthopnea and a jugular venous pressure $<8$ $\mathrm{mmHg}$ at discharge, the risk for readmission, death, or unplanned hospital visits within 60 days was 50\% [57]. This suggests that clinical assessments are insufficient to detect euvolemia. A retrospective analysis of the CardioMEMS Heart Sensor Allows Monitoring of Pressure to Improve Outcomes in NYHA Class III Patients (CHAMPION) trial indicated that patients in the clinical arm were deemed optivolemic at much higher filling pressures than patients in the hemodynamic guided arm, inevitably resulting in higher readmission rates [58]. Testani et al. [59] showed that patients who achieved hemoconcentration during AHF treatment had significantly better prognoses, even if WRF developed [59-62]. Hemoconcentration occurs when both the interstitium and plasma compartment are free of excess volume, and additional diuresis will not result in the interstitium refilling the plasma compartment [61]. However, whether hemoconcentration can be used to guide decongestion has not been tested, and the intrinsic fluctuations in hemoglobin concentrations may be problematic. Further studies are needed to determine when a congested patient with volume overload is dry [63].

\section{Tackling congestion with volume redistribution}

When volume redistribution causes congestion, therapy aims to enhance venous capacitance function and lower cardiac filling pressures [64]. To achieve this, a combination of vasodilators and low doses of intravenous diuretics are used. Intravenous vasodilators reduce preload by venodilation; thereby, enhancing venous capacitance; they also induce arteriolar vasodilation, which reduces the afterload on the failing heart [65], allowing the heart to activate the Frank-Starling mechanism with a drop in left ventricular end-diastolic pressure and a parallel increase in stroke volume. Initial bed rest can also enhance the effects of intravenous dilatory drugs [66]. 
Central filling pressures often drop spontaneously following right heart catheterization and the patient's admission to a cardiac care unit, even before any vasoactive drugs have been administered [50]. This may be due to a reduction in adrenergic stress, which enhances venous compliance. Although the ESCAPE trial did not demonstrate the benefit of targeting filling pressures (goal: right atrial pressure $<8 \mathrm{mmHg}$; pulmonary capillary wedge pressure $<15 \mathrm{mmHg}$ ), lower filling pressures in patients awaiting heart transplants are associated with improved outcomes $[35,67,68]$. Similar findings were documented in the CHAMPION trial [58]. Numerous vasodilatory agents are being developed that improve venous compliance and capacitance and target systemic vascular resistance, with secondary improvements in cardiac output and pulmonary capillary wedge pressure. These novel drugs include cenderitide, $\beta$-arrestin-biased angiotensin II type 1-receptor blockers, nitroxyl-donors, soluble guanylate cyclase modulators, and nicorandil [69]. However, several recently developed vasodilators failed to improve clinical outcome in heart failure patients $[10,70]$. Once filling pressures have been normalized, tapering of intravenous vasodilatory drugs should be accompanied by uptitration of neurohumoral blockers [71]. Refractory or recurrent volume misdistribution is often caused by poor intrinsic cardiac function. Increased cardiac filling pressures activate the venous system to meet the preload demand. Patients should be meticulously evaluated to determine their suitability for advanced therapies including cardiac resynchronization therapy, left ventricular assist devices, and heart transplants [64]. Because the risk of recurrence is related to the total duration of high filling pressures, monitoring filling pressures in these patients is particularly important [72].

\section{Preventing recurrence of congestion}

In addition to achieving decongestion, one of the most effective interventions is an appropriate discharge policy. Titration of neurohormonal blockers is essential. These include $\beta$-blockers, angiotensin-converting enzyme (ACE) inhibitors, angiotensin receptor blockers, and angiotensin receptor/neprilysin inhibitors. The discharge policy should ensure that ambulatory rehabilitation is initiated and a low salt diet with fluid restriction is implemented. In addition, a stable dose of oral diuretics must be formulated, and follow-up procedures should be in place to monitor the patient [73]. In the OPTIMIZE-HF (Organized Program to Initiate Lifesaving Treatment in Hospitalized Patients with Heart Failure) trial, treatments with $\beta$-blockers and ACE-inhibitors were strongly associated with better postdischarge outcomes [74]. A recent analysis also found that achieving decongestion and uptitrating ACE-inhibition has a synergistic effect [75], suggesting that therapies should be optimized while treatment for decongestion is ongoing.

\section{CONCLUSIONS}

Improving our understanding of the pathophysiology of congestion allows us to explain why congestion at discharge results in a poor prognosis for AHF patients. Increased filling pressures are not always associated with volume excesses. A careful evaluation of the congested patient is necessary to determine whether volume overload or volume misdistribution is primarily responsible for congestion. In both cases, achieving complete decongestion is the target. If volume overload is present, well-designed diuretic therapies may be necessary. In patients with volume misdistribution, a combination of vasodilators and low-dose diuretics may be implemented. Once decongestion has been achieved, every effort should be made to prevent congestion recurring. A well-designed heart-failure-care program, emphasizing neurohormonal-blocker uptitration, rehabilitation, follow-up procedures, the treatment of comorbidities, device-based therapies, sodium restriction, and education can reduce the risk for recurrence.

\section{Conflict of interest}

No potential conflict of interest relevant to this article was reported.

\section{Acknowledgments}

Pieter Martens is supported by a doctoral fellowship by the Research Foundation-Flanders (FWO, grant-num-

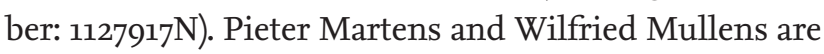
researchers for the Limburg Clinical Research Program (LCRP) UHasselt-ZOL-Jessa, supported by the foundation Limburg Sterk Merk (LSM), Hasselt University, Ziekenhuis Oost-Limburg and Jessa Hospital. 


\section{REFERENCES}

1. Gheorghiade M, Pang PS. Acute heart failure syndromes. J Am Coll Cardiol 2009;53:557-573.

2. Adams KF Jr, Fonarow GC, Emerman CL, et al. Characteristics and outcomes of patients hospitalized for heart failure in the United States: rationale, design, and preliminary observations from the first 100,000 cases in the Acute Decompensated Heart Failure National Registry (ADHERE). Am Heart J 2005;149:209-216.

3. Go AS, Mozaffarian D, Roger VL, et al. Heart disease and stroke statistics: 2013 update. A report from the American Heart Association. Circulation 2013;127:e6-e245.

4. Fang J, Mensah GA, Croft JB, Keenan NL. Heart failure-related hospitalization in the U.S., 1979 to 2004. J Am Coll Cardiol 2008;52:428-434.

5. Cotter G, Kaluski E, Stangl K, et al. The hemodynamic and neurohormonal effects of low doses of tezosentan (an endothelin A/B receptor antagonist) in patients with acute heart failure. Eur J Heart Fail 2004;6:601-609.

6. Giamouzis G, Butler J, Starling RC, et al. Impact of dopamine infusion on renal function in hospitalized heart failure patients: results of the Dopamine in Acute Decompensated Heart Failure (DAD-HF) Trial. J Card Fail 2010;16:922-930.

7. Konstam MA, Gheorghiade M, Burnett JC Jr, et al. Effects of oral tolvaptan in patients hospitalized for worsening heart failure: the EVEREST Outcome Trial. JAMA 2007;297:1319-1331.

8. Massie BM, O'Connor CM, Metra M, et al. Rolofylline, an adenosine A1-receptor antagonist, in acute heart failure. N Engl J Med 2010;363:1419-1428.

9. McMurray JJ, Teerlink JR, Cotter G, et al. Effects of tezosentan on symptoms and clinical outcomes in patients with acute heart failure: the VERITAS randomized controlled trials. JAMA 2007;298:2009-2019.

10. O'Connor CM, Starling RC, Hernandez AF, et al. Effect of nesiritide in patients with acute decompensated heart failure. N Engl J Med 2011;365:32-43.

11. McMurray JJ, Adamopoulos S, Anker SD, et al. ESC guidelines for the diagnosis and treatment of acute and chronic heart failure 2012: the task force for the diagnosis and treatment of acute and chronic heart failure 2012 of the European Society of Cardiology. Developed in collaboration with the Heart Failure Association (HFA) of the ESC. Eur J Heart Fail 2012;14:803-869.
12. Yancy CW, Jessup M, Bozkurt B, et al. 2013 ACCF/AHA guideline for the management of heart failure: a report of the American College of Cardiology Foundation/American Heart Association Task Force on Practice Guidelines. J Am Coll Cardiol 2013;62:e147-e239.

13. Kociol RD, McNulty SE, Hernandez AF, et al. Markers of decongestion, dyspnea relief, and clinical outcomes among patients hospitalized with acute heart failure. Circ Heart Fail 2013;6:240-245.

14. Mentz RJ, O'Connor CM. Pathophysiology and clinical evaluation of acute heart failure. Nat Rev Cardiol 2016;13:28-35.

15. Zile MR, Bennett TD, St John Sutton M, et al. Transition from chronic compensated to acute decompensated heart failure: pathophysiological insights obtained from continuous monitoring of intracardiac pressures. Circulation 2008;118:1433-1441.

16. Mullens W, Verbrugge FH, Nijst P, Tang WHW. Renal sodium avidity in heart failure: from pathophysiology to treatment strategies. Eur Heart J 2017;38:1872-1882.

17. Nijst P, Verbrugge FH, Grieten L, et al. The pathophysiological role of interstitial sodium in heart failure. J Am Coll Cardiol 2015;65:378-388.

18. Gelman S. Venous function and central venous pressure: a physiologic story. Anesthesiology 2008;108:735-748.

19. Greenway CV. Role of splanchnic venous system in overall cardiovascular homeostasis. Fed Proc 1983;42:16781684.

20. Miller WL, Mullan BP. Understanding the heterogeneity in volume overload and fluid distribution in decompensated heart failure is key to optimal volume management: role for blood volume quantitation. JACC Heart Fail 2014;2:298-305

21. Chaudhry SI, Wang Y, Concato J, Gill TM, Krumholz HM. Patterns of weight change preceding hospitalization for heart failure. Circulation 2007;116:1549-1554.

22. Verbrugge FH, Dupont M, Steels P, et al. Abdominal contributions to cardiorenal dysfunction in congestive heart failure. J Am Coll Cardiol 2013;62:485-495.

23. Androne AS, Hryniewicz K, Hudaihed A, Mancini D, Lamanca J, Katz SD. Relation of unrecognized hypervolemia in chronic heart failure to clinical status, hemodynamics, and patient outcomes. Am J Cardiol 2004;93:1254-1259.

24. Greenway CV, Lister GE. Capacitance effects and blood reservoir function in the splanchnic vascular bed during non-hypotensive haemorrhage and blood volume expan- 
sion in anaesthetized cats. J Physiol 1974;237:279-294.

25. Fallick C, Sobotka PA, Dunlap ME. Sympathetically mediated changes in capacitance: redistribution of the venous reservoir as a cause of decompensation. Circ Heart Fail 2011;4:669-675.

26. Davis KL, Laine GA, Geissler HJ, Mehlhorn U, Brennan M, Allen SJ. Effects of myocardial edema on the development of myocardial interstitial fibrosis. Microcirculation 2000;7:269-280.

27. Desai KV, Laine GA, Stewart RH, et al. Mechanics of the left ventricular myocardial interstitium: effects of acute and chronic myocardial edema. Am J Physiol Heart Circ Physiol 2008;294:H2428-H2434.

28. Davis KL, Mehlhorn U, Laine GA, Allen SJ. Myocardial edema, left ventricular function, and pulmonary hypertension. J Appl Physiol (1985) 1995;78:132-137.

29. Mullens W, Abrahams Z, Francis GS, et al. Importance of venous congestion for worsening of renal function in advanced decompensated heart failure. J Am Coll Cardiol 2009;53:589-596.

30. Damman K, van Deursen VM, Navis G, Voors AA, van Veldhuisen DJ, Hillege HL. Increased central venous pressure is associated with impaired renal function and mortality in a broad spectrum of patients with cardiovascular disease. J Am Coll Cardiol 2009;53:582-588.

31. Nijst P, Martens P, Dupont M, Tang WHW, Mullens W. Intrarenal flow alterations during transition from euvolemia to intravascular volume expansion in heart failure patients. JACC Heart Fail 2017;5:672-681.

32. Adamson PB, Smith AL, Abraham WT, et al. Continuous autonomic assessment in patients with symptomatic heart failure: prognostic value of heart rate variability measured by an implanted cardiac resynchronization device. Circulation 2004;110:2389-2394.

33. Mullens W, Abrahams Z, Skouri HN, et al. Elevated intra-abdominal pressure in acute decompensated heart failure: a potential contributor to worsening renal function? J Am Coll Cardiol 2008;51:300-306.

34. Colombo PC, Doran AC, Onat D, et al. Venous congestion, endothelial and neurohormonal activation in acute decompensated heart failure: cause or effect? Curr Heart Fail Rep 2015;12:215-222.

35. Binanay C, Califf RM, Hasselblad V, et al. Evaluation study of congestive heart failure and pulmonary artery catheterization effectiveness: the ESCAPE trial. JAMA 2005;294:1625-1633.
36. Drazner MH, Hamilton MA, Fonarow G, Creaser J, Flavell C, Stevenson LW. Relationship between right and left-sided filling pressures in 1000 patients with advanced heart failure. J Heart Lung Transplant 1999;18:1126-1132.

37. Capomolla S, Ceresa M, Pinna G, et al. Echo-Doppler and clinical evaluations to define hemodynamic profile in patients with chronic heart failure: accuracy and influence on therapeutic management. Eur J Heart Fail 2005;7:624630.

38. Rohde LE, Palombini DV, Polanczyk CA, Goldraich LA, Clausell N. A hemodynamically oriented echocardiography-based strategy in the treatment of congestive heart failure. J Card Fail 2007;13:618-625.

39. Francis GS, Felker GM, Tang WH. A test in context: critical evaluation of natriuretic peptide testing in heart failure. J Am Coll Cardiol 2016;67:330-337.

40. Verbrugge FH, Grieten L, Mullens W. New insights into combinational drug therapy to manage congestion in heart failure. Curr Heart Fail Rep 2014;11:1-9.

41. Guyton AC, Prather J, Scheel K, McGehee J. Interstitial fluid pressure. IV. Its effect on fluid movement through the capillary wall. Circ Res 1966;19:1022-1030.

42. ter Maaten JM, Valente MA, Damman K, Hillege HL, Navis G, Voors AA. Diuretic response in acute heart failure-pathophysiology, evaluation, and therapy. Nat Rev Cardiol 2015;12:184-192.

43. Fonarow GC, Corday E; ADHERE Scientific Advisory Committee. Overview of acutely decompensated congestive heart failure $(\mathrm{ADHF})$ : a report from the ADHERE registry. Heart Fail Rev 2004;9:179-185.

44. Felker GM, Lee KL, Bull DA, et al. Diuretic strategies in patients with acute decompensated heart failure. N Engl J Med 2011;364:797-805.

45. Matsue Y, Damman K, Voors AA, et al. Time-to-furosemide treatment and mortality in patients hospitalized with acute heart failure. J Am Coll Cardiol 2017;69:30423051.

46. Martens P, Verbrugge FH, Nijst P, Dupont M, Mullens W. Changes in loop diuretic dose and outcome after cardiac resynchronization therapy in patients with heart failure and reduced left ventricular ejection fractions. Am J Cardiol 2017;120:267-273.

47. Jentzer JC, DeWald TA, Hernandez AF. Combination of loop diuretics with thiazide-type diuretics in heart failure. J Am Coll Cardiol 2010;56:1527-1534.

48. Kim GH. Long-term adaptation of renal ion transporters 
to chronic diuretic treatment. Am J Nephrol 2004;24:595605 .

49. Verbrugge FH, Steels P, Grieten L, Nijst P, Tang WH, Mullens W. Hyponatremia in acute decompensated heart failure: depletion versus dilution. J Am Coll Cardiol 2015;65:480-492.

50. Martens P, Nijst P, Mullens W. Current approach to decongestive therapy in acute heart failure. Curr Heart Fail Rep 2015;12:367-378.

51. Butler J, Anstrom KJ, Felker GM, et al. Efficacy and safety of spironolactone in acute heart failure: the ATHENA-HF randomized clinical trial. JAMA Cardiol 2017;2:950-958.

52. Verbrugge FH, Dupont M, Bertrand PB, et al. Determinants and impact of the natriuretic response to diuretic therapy in heart failure with reduced ejection fraction and volume overload. Acta Cardiol 2015;70:265-273.

53. Martens P, Mathieu C, Verbrugge FH. Promise of SGLT2 inhibitors in heart failure: diabetes and beyond. Curr Treat Options Cardiovasc Med 2017;19:23.

54. Neal B, Perkovic V, Mahaffey KW, et al. Canagliflozin and cardiovascular and renal events in type 2 diabetes. N Engl J Med 2017;377:644-657.

55. Zinman B, Wanner C, Lachin JM, et al. Empagliflozin, cardiovascular outcomes, and mortality in type 2 diabetes. N Engl J Med 2015;373:2117-2128.

56. Costanzo MR, Ronco C, Abraham WT, et al. Extracorporeal ultrafiltration for fluid overload in heart failure: current status and prospects for further research. J Am Coll Cardiol 2017;69:2428-2445.

57. Lala A, McNulty SE, Mentz RJ, et al. Relief and recurrence of congestion during and after hospitalization for acute heart failure: insights from Diuretic Optimization Strategy Evaluation in Acute decompensated Heart Failure (DOSE-AHF) and Cardiorenal Rescue Study in acute decompensated Heart Failure (CARESS-HF). Circ Heart Fail 2015;8:741-748.

58. Costanzo MR, Stevenson LW, Adamson PB, et al. Interventions linked to decreased heart failure hospitalizations during ambulatory pulmonary artery pressure monitoring. JACC Heart Fail 2016;4:333-344.

59. Testani JM, Chen J, McCauley BD, Kimmel SE, Shannon RP. Potential effects of aggressive decongestion during the treatment of decompensated heart failure on renal function and survival. Circulation 2010;122:265-272.

6o. Greene SJ, Gheorghiade M, Vaduganathan M, et al. Haemoconcentration, renal function, and post-discharge out- comes among patients hospitalized for heart failure with reduced ejection fraction: insights from the EVEREST trial. Eur J Heart Fail 2013;15:1401-1411.

61. Testani JM, Brisco MA, Chen J, McCauley BD, Parikh CR, Tang WH. Timing of hemoconcentration during treatment of acute decompensated heart failure and subsequent survival: importance of sustained decongestion. J Am Coll Cardiol 2013;62:516-524.

62. van der Meer P, Postmus D, Ponikowski P, et al. The predictive value of short-term changes in hemoglobin concentration in patients presenting with acute decompensated heart failure. J Am Coll Cardiol 2013;61:1973-1981.

63. Katz SD. In search of euvolemia in heart failure. JACC Heart Fail 2014;2:306-307.

64. Ponikowski P, Voors AA, Anker SD, et al. 2016 ESC guidelines for the diagnosis and treatment of acute and chronic heart failure. Kardiol Pol 2016;74:1037-1147.

65. Mullens W, Abrahams Z, Francis GS, et al. Sodium nitroprusside for advanced low-output heart failure. J Am Coll Cardiol 2008;52:200-207.

66. On bedresting in heart failure. Lancet 1990;336:975-976.

67. Lucas C, Johnson W, Hamilton MA, et al. Freedom from congestion predicts good survival despite previous class IV symptoms of heart failure. Am Heart J 2000;140:840847.

68. Morley D, Brozena SC. Assessing risk by hemodynamic profile in patients awaiting cardiac transplantation. Am J Cardiol 1994;73:379-383.

69. Singh A, Laribi S, Teerlink JR, Mebazaa A. Agents with vasodilator properties in acute heart failure. Eur Heart J 2017;38:317-325.

70. Packer M, O'Connor C, McMurray JJV, et al. Effect of ularitide on cardiovascular mortality in acute heart failure. $\mathrm{N}$ Engl J Med 2017;376:1956-1964.

71. Verbrugge FH, Dupont M, Finucan M, et al. Response and tolerance to oral vasodilator up-titration after intravenous vasodilator therapy in advanced decompensated heart failure. Eur J Heart Fail 2015;17:956-963.

72. Stevenson LW, Zile M, Bennett TD, et al. Chronic ambulatory intracardiac pressures and future heart failure events. Circ Heart Fail 2010;3:580-587.

73. Gheorghiade M, Vaduganathan M, Fonarow GC, Bonow RO. Rehospitalization for heart failure: problems and perspectives. J Am Coll Cardiol 2013;61:391-403.

74. Fonarow GC, Abraham WT, Albert NM, et al. Association between performance measures and clinical out- 
comes for patients hospitalized with heart failure. JAMA 2007;297:61-70.

75. Kula AJ, Hanberg JS, Wilson FP, et al. Influence of titra- tion of neurohormonal antagonists and blood pressure reduction on renal function and decongestion in decompensated heart failure. Circ Heart Fail 2016;9:eoo2333. 